\title{
COHOMOLOGY OF FINITE ABELIAN $p$-GROUPS AND FREE ACTIONS
}

\author{
KAHTAN H. ALZUBAIDY
}

\begin{abstract}
The generators of the integral cohomology ring $H^{*}(G, \mathbb{Z})$ of a finite abelian $p$-group $G$ have been constructed and an application to free action of a finite group on a product of spheres $\left(S^{n}\right)^{k}$ has been given.
\end{abstract}

\section{Introduction}

In this paper Bockestein homomorphism is used to work out the generators and some basic relations of the integral cohomology ring $H^{*}(G, \mathbb{Z})$ of an abelian $p$-group $G=\mathbb{Z}_{p^{t_{1}}} \times \mathbb{Z}_{p^{t_{2}}} \times \cdots \times \mathbb{Z}_{p^{t_{r}}}$, aproduct of cyclic groups of orders powers of the prime $p$. An application is also given about the maximum possible rank for a $p$-group to act freely, preserving orientation, on $\left(S^{n}\right)^{k}, k \geq 2$, a product of $k$ spheres of dimension $n$.

\section{Preleminaries}

Let $0 \rightarrow \mathbb{Z} \stackrel{p}{\rightarrow} \mathbb{Z}^{j} \stackrel{\text { Z}}{\rightarrow} \rightarrow 0$ be a short exact sequence, where the first map $p$ is defined by multiplication by the prime $p$ and second map $j$ is by taking modulo $p$. The Bockestein homomorphism $\Delta: H^{q}\left(G, \mathbb{Z}_{p}\right) \rightarrow H^{q+1}(G, \mathbb{Z})$ is the connecting homomorphism. The following sequence is however exact:

$$
0 \rightarrow H^{q}(G, \mathbb{Z}) \stackrel{j_{*}}{\longrightarrow} H^{q}\left(G, \mathbb{Z}_{p}\right) \stackrel{\delta}{\longrightarrow} H^{q+1}(G, \mathbb{Z}) \rightarrow 0 .
$$

$j_{*}$ is monomorphism . Therefore $H^{*}(G, Z)=\operatorname{ker} \delta=\operatorname{ker} \Delta$. Observe that the composite $\Delta \Delta=j_{*} \delta j_{*} \delta=j_{*} 0 \delta=0$.

\section{Computations}

$H^{*}\left(\mathbb{Z}_{p^{t}}, \mathbb{Z}_{p}\right)=P[v] \otimes E[u]$, tensor product of polynomial and exterior algebras, where $\operatorname{deg} v=2, \operatorname{deg} u=1, p^{t} v=p^{t} u=0$ and $\Delta u=v$. That is if $p \geq 3$. When $p=2$, Received January 28, 2010. 2000 Mathematics Subject Classification. 20J06: cohomology of groups and 57S25: groups acting on special manifolds.

Key words and phrases. Cohomology of groups, free actions. 
$H^{*}\left(\mathbb{Z}_{p^{t}}, \mathbb{Z}_{p}\right)=P[v]$, where $\operatorname{deg} v=1, p^{t} v=0$ and $\Delta v=v^{2}[4]$, Let $G$ be a finite abelian $p$-group. Then $G=\mathbb{Z}_{p^{t_{1}}} \times \mathbb{Z}_{p^{t_{2}}} \times \cdots \times \mathbb{Z}_{p^{t_{r}}}$, a product of cyclic groups of orders powers of the prime $p$. Without lost of generality, we can assume $t_{1} \leq t_{2} \leq \cdots \leq t_{r}$. By Kunneth formula [3] we have

$$
H^{*}\left(G, \mathbb{Z}_{p}\right) \cong H^{*}\left(\mathbb{Z}_{p^{t_{1}}}, \mathbb{Z}_{p}\right) \otimes H^{*}\left(\mathbb{Z}_{p^{t_{2}}}, \mathbb{Z}_{p}\right) \otimes \cdots \otimes H^{*}\left(\mathbb{Z}_{p^{t_{r}}}, \mathbb{Z}_{p}\right)
$$

Suppose that $p \geq 3$. Then $H^{*}\left(G, \mathbb{Z}_{p}\right) \cong P\left[v_{1}, v_{2}, \ldots, v_{r}\right] \otimes E\left[u_{1}, u_{2}, \ldots, u_{r}\right]$ where $\operatorname{deg} v_{i}=2, \operatorname{deg} u_{i}=1, p^{t_{i}} v_{i}=p^{t_{i}} u_{i}=0$ and $\Delta u_{i}=v_{i}(i=1,2, \ldots, n)$. As an operator $\Delta$ has the property $\Delta(a b)=(\Delta a) b+(-1)^{\operatorname{deg} a} a(\Delta b)$. The product of these generators satisfies the anticommutative rule $a b=(-1)^{\operatorname{deg} a \operatorname{deg} b} b a$. In particular $u_{i} u_{j}=-u_{j} u_{i}$ if $i \neq j$ and $u_{i}^{2}=0$. Multiplication by $v_{i}$ is commutative. $\Delta \Delta=0$. Therefore $\Delta v_{i}=0$. The generators of $H^{*}(G, \mathbb{Z})$ are the elements of ker $\Delta$. They are given as follows:

$$
\sum_{k=1}^{j}(-1)^{i_{k}} u_{i_{1}} u_{i_{2}} \ldots \hat{u}_{i_{k}} \ldots u_{i_{j}} v_{i_{k}} \quad \text { where } i, j \in\{1,2, \ldots, r\} \text {. }
$$

The number of such generators is $\left(\begin{array}{l}r \\ j\end{array}\right)$ for each $j$. Suppose now $p=2$. Then $H^{*}\left(G, \mathbb{Z}_{p}\right) \cong$ $P\left[v_{1}, v_{2}, \ldots, v_{r}\right]$ where $\operatorname{deg} v_{i}=1, p^{r_{i}} v_{i}=0$ and $\Delta v_{i}=v_{i}^{2}(i=1,2, \ldots, n)$.

Thus the generators of $H^{*}(G, \mathbb{Z})$ are given as follows:

$$
\sum_{k=1}^{j} v_{i_{1}} v_{i_{2}} \ldots v_{i_{k}}^{2} \ldots v_{i_{j}} \quad \text { where } i, j \in\{1,2, \ldots, r\} .
$$

They are also $\left(\begin{array}{l}r \\ j\end{array}\right)$ generators for each $j$. In each case there are $2^{r}-1$ generators. Thus we have

Theorem. The integeral cohomology ring $H^{*}(G, \mathbb{Z})$ of an abelian p-group $G=\mathbb{Z}_{p^{t_{1}}} \times$ $\mathbb{Z}_{p^{t_{2}}} \times \cdots \times \mathbb{Z}_{p^{t_{r}}}$ is generated by $\alpha_{k}^{(d)} ; d=2, \ldots, r+1 ; k=1, \ldots,\left(\begin{array}{c}r \\ d-1\end{array}\right)$ where $\operatorname{deg} \alpha_{k}^{(d)}=d$ and its additive order is $p^{t_{k}}$. Further more $\left(\alpha_{k}^{d)}\right)^{2}=0$ if $d$ is odd.

Corollary. The exponent of $H^{*}(G, \mathbb{Z})$ is $p^{t_{r}}$.

\section{Special Cases.}

$H^{*}\left(\mathbb{Z}_{p^{t}}, \mathbb{Z}\right)$ is generated by $\alpha$ where $\operatorname{deg} \alpha=2$ and $p^{t} \alpha=0$. $\quad H^{*}\left(\mathbb{Z}_{p^{s}} \times \mathbb{Z}_{p^{t}}, \mathbb{Z}\right)$ where $s \leq t$ is generated by $\alpha, \beta$ and $\mu$ where $\operatorname{deg} \alpha=\operatorname{deg} \beta=2$ and $\operatorname{deg} \mu=3$ and $p^{s} \alpha=p^{t} \beta=p^{s} \mu=0$ and $\mu^{2}=0$ if $p \geq 3 . \mu^{2}=\alpha^{2} \beta+\alpha \beta^{2}$ when $p=2$.

Finally $H^{*}\left(\left(\mathbb{Z}_{p}\right)^{3}, \mathbb{Z}\right)$ is generated by $\alpha, \beta, \gamma, \mu, \nu, \chi, \xi$ where $\operatorname{deg} \alpha=\operatorname{deg} \beta=\operatorname{deg} \gamma=$ $2, \operatorname{deg} \mu=\operatorname{deg} \nu=\operatorname{deg} \chi=3, \operatorname{deg} \xi=4$. There are two different sets of relations for $p=2$ and $p \geq 3[4]$. 


\section{Application}

W. Browder [2] proved that when $n$ is odd and $p$ a prime if $\left(\mathbb{Z}_{p}\right)^{r}$ acts freely, preserving orientation, on $\left(S^{n}\right)^{k}$ with trivial action on the homology, then $r \leq k$. This gives the limitation on the rank $r$ when any finite group acts freely on $\left(S^{n}\right)^{k}$. A different but simpler proof to this result is now introduced.

By [1, Proposition 1], the following sequence is exact

$$
0 \rightarrow H^{m}(G, \mathbb{Z}) \rightarrow H^{n+1}(G, \mathbb{Z}) \rightarrow\left(\mathbb{Z}_{|G|}\right)^{k} \rightarrow H^{m+1}(G, \mathbb{Z}) \rightarrow H^{n}(G, \mathbb{Z}) \rightarrow 0
$$

where $m=(k-1) n-k+2 . m$ is also odd. Observe that $H^{n+1}(G, \mathbb{Z})|=| G|| H^{m}(G, \mathbb{Z}) \mid$ and $|G|=p^{r}$. From the structure of $H^{*}(G, \mathbb{Z})$ we have $p^{r} \leq\left|H^{n+1}(G, \mathbb{Z})\right|$, since $n+1$ is even and $H^{m}(G, \mathbb{Z}) \mid \leq p^{k-r}$, since $m=(n-1)(k-1)+1$ is odd and $n-1$ is even. Then $p^{r} \leq p^{r} p^{k-r}$. Thus $r \leq k$.

The author thanks the referee for several useful comments.

\section{References}

[1] K. H. Alzubaidy, Free actions on $\left(S^{n}\right)^{k}$, Mathematika, 32(1985), 49-54.

[2] W. Browder, Cohomology and group action, Invent. Math., 71(1983), 599-607.

[3] K. S. Brown, Cohomology and Groups, Springer-Verlag, New York, 1982.

[4] G. Lewis, The integral cohomology rings of groups of order $p^{3}$, Trans. Amer. Math. Soc. 132(1968), 501-529.

Department of Mathematics, Science Faculty, Garyounis University, Benghazi, Libya.

E-mail: kahtanalzubaidy@yahoo.com 